\title{
An unusual find in sputum: Exoskeleton of Pediculus capitis
}

\author{
Jauhari S*, Nautiyal S, Roy R C, Mahawal B S, Kataria V K \\ Department of Microbiology, SGRRIM\&HS, Dehradun (Uttarakhand)
}

\begin{abstract}
In the present case of chronic cough, sputum sample was sent to laboratory to rule out fungal etiology. An interesting structure was observed on $10 \% \mathrm{KOH}$ preparation and was categorized as exoskeleton of nymphal stage of Pediculus capitis. Though an artifact, a literature search revealed no such observation so far from sputum.
\end{abstract}

Key words: sputum examination, exoskeleton of Pediculus capitis

\section{Introduction}

Chronic pulmonary tuberculosis poses a global health emergency. More than 5.8 million new cases of TB (all forms, both pulmonary and extrapulmonary) were reported to the WHO in 2009: 95\% of cases were reported from developing countries [1]. Sputum examination is one of the basic and fundamental tests for diagnosing tuberculosis. Overall sensitivity of smear has been reported to range from 22-80\% [2].

In developing country like India, in the low socioeconomic strata the infestations with ectoparasites like head lice are very common owing to poor hygiene and overcrowding in their dwellings. Moreover, the increase in prevalence of head lice during the 1990s, research interest in human louse infestation is now greater than at any time since World War I [3].

\section{Case report}

A 68 year old female presented in medicine OPD with chief complaints of low grade fever, difficulty in breathing and brief spells of apneas which were off and on for last 3 months. Patient was a farmer by occupation and belonged to a low socio economic status. She was a habitual smoker (beedi) for the past 20 years, anaemic and suffered from diabetes but denied taking any regular treatment for the same. There was no history of pulmonary tuberculosis, other prolonged respiratory illness or immunosuppressive therapy in the past.

The family history revealed that her husband was undergoing anti-tubercular treatment for the past one month. She accepted that she took the treatment for her complaints at her native village from a quack but could not get any relief. As suggested by her husband and some of her neighbours, she came to this tertiary care centre at Dehradun for further evaluation and treatment.

Physical examination revealed that the patient was conscious, afebrile and lethargic. She was having a respiratory rate of 24 breaths/ min. Her blood pressure was 142/80 mmHg. No adventitious chest sounds were heard. Patient was advised to undergo certain laboratory investigations for diagnostic evaluation.

Complete blood count revealed: WBC- 9.7 cells $/ \mathrm{mm}^{3}$, with differential count of $48 \%$ Neutrophils, $38 \%$ Lymphocytes, 4\% Monocytes and 10\% Eosinophils. Her hemoglobin level was $10.6 \mathrm{gm} / \mathrm{dl}$ and her platelet count was 490 cells $/ \mathrm{mm}^{3}$. ESR was $30 \mathrm{~mm} / \mathrm{hr}$. Her renal and liver functions were within normal range.

Rapid tests for HIV, HBV and HCV were negative. A chest radiograph revealed mild opacities in the lower lobe of right lung. There was no pleural or pericardial effusion. Cardiac shadows were normal [ Fig. 1]. Blood cultures were also sent but no organism was isolated.

Following these examinations, patient's sputum was also sent for Gram staining, Ziehl Neelsen (ZN) staining for acid fast bacilli, culture for Mycobacterium tuberculosis and $\mathrm{KOH}$ mount for fungal elements. ZN staining of the smear revealed presence of acid fast bacilli suggestive of Mycobacterium tuberculosis and the smear was graded as $2+$ according to RNTCP.

On examination of the $\mathrm{KOH}$ mount of sputum for fungal elements, no fungal elements were seen though it revealed a unique structure. This structure resembled the exoskeleton of head louse but could not qualify for the size of head louse [Fig. 2]. A fresh sputum sample was repeated and examined to clarify our doubts regarding this unusual finding. The second sample was negative for the same. We could not find anything resembling this finding even in the published data. 


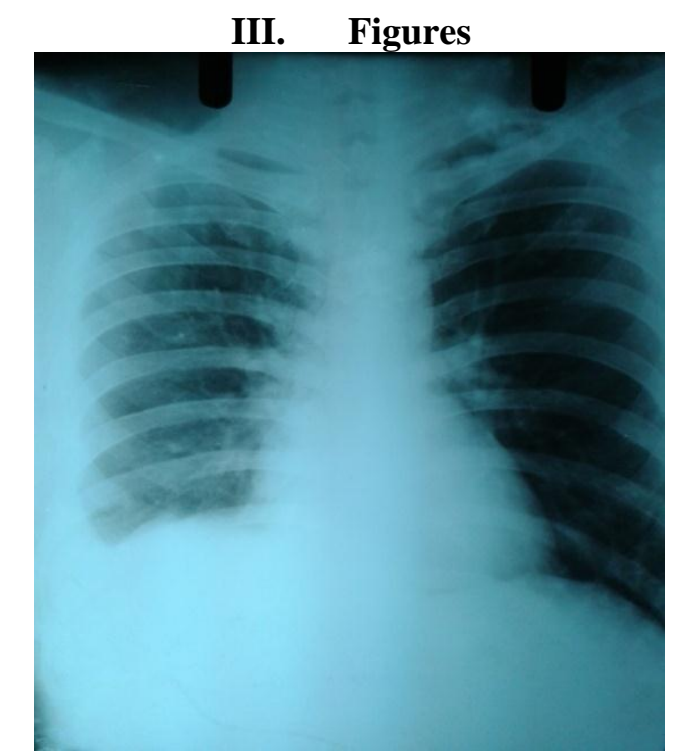

Fig.1. X-ray chest showing opacity in right lower lobe

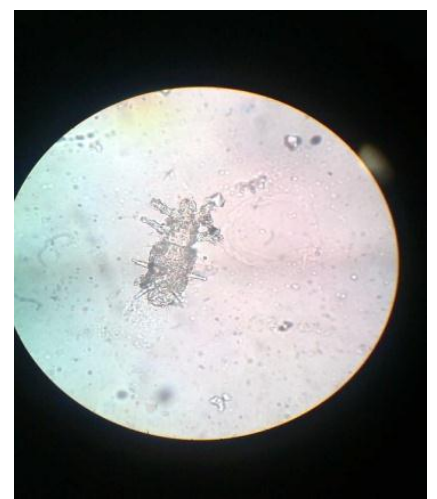

Fig.2: Exoskeleton of Pediculus capitis

\section{Discussion}

Lice are parasites of warm-blooded animals, including man. The three species of lice that parasitize humans are the head louse, body louse, and pubic (crab) louse. All three suck blood and cause considerable itching when they feed or crawl on the body. Head lice are not known to transmit any human pathogens. Skin irritation at the feeding site, secondary bacterial infections from scratching, and the psychological "trauma" of the infestations are the chief human health concerns associated with head lice.

Head lice are members of a group of insects (Order Pthiraptera, Suborder Anoplura, Family Pediculidae) that are ectoparasites of birds or mammals. Pediculidae have been associated with humans since antiquity. Most experts consider the human head louse and human body louse to be variants of the same species (Pediculus humanus) that segregate by habitat on the host. Lice are host-specific, so lice found on humans will not survive on other animal hosts and vice versa.

Human head lice are minute, wingless insects that are obligate ectoparasites. They are small in size, about $1-5 \mathrm{~mm}$ or $1 / 32$ to $3 / 16$ of an inch in length, and either light gray or dark

coloured in appearance, the latter due to ingested blood. They are flattened dorso-ventrally and have six jointed legs with specially adapted claws for holding onto hair.

Treatment for head lice is recommended for persons diagnosed with an active infestation. All household members and other close contacts should be checked; those persons with evidence of an active infestation should be treated. Some experts believe prophylactic treatment is prudent for persons who share the same bed with actively-infested individuals. All infested persons (household members and close contacts) and their bedmates should be treated at the same time.[4]

A detailed study regarding the life cycle of head lice revealed that there are 3 nymphal stages punctuated by moulting during the development of head lice from eggs to adult form. Each of these nymphal stages increases the size of head lice without any change in its structure and it looks like a miniature adult $[4,5]$. 
In the present specimen, since it was only a hollow structure without life, visible microscopically only, we derived that it was just an aerial contamination by moulted exoskeleton of nymphal stage of head lice during collection of the sputum. It was interesting to note that the patient herself was harbouring this ectoparasite.

Treatment regarding head lice was also suggested to the lady and her family members but on follow up it was observed that she was still harbouring the parasite. Clinical history revealed that all the family members had also taken the prescribed topical application for lice infestation.

Clinicoepidemiological studies are urgently needed to identify the factors which govern the emergence and spread of strains of $P$ humanus capitis that are resistant to insecticide or acaricide. In this case though we did not evaluate the resistance of this strain of head louse to acaricides, but different patterns of resistances have been reported [6].

She was later on followed with anti-tubercular treatment at DOTS centre and is responding well to the treatment.

\section{References}

[1]. Longo, Fauci, Kasper, Hauser, Jameson and Loscolzo: Harrison's Principle of Internal Medicine $18^{\text {th }}$ ed, Vol 1. Mc Graw Hill Medical ;2012, p.1340

[2]. Lipsky, B.A., J.Gates, F.C.Tenover, J.J.Plorde. Factors affecting the clinical value of microscopy for acid fast bacilli. Rev. Infect. Dis. $1984 ; 6: 214-222$

[3]. Burgress IF. Current treatments for pediculosis capitis. Curr Opin Infect Dis 2009; 22: 131-136

[4]. Georgia Department of Public Health; Head Lice Manual; pages 5-7; pdf on internet accessed on 15/3/2013

[5]. A H Suryakantha, Community Medicine with Recent Advances (second edition); Jaypee Brothers Medical Publishers (P) LTD;2010; pages 137-139.

[6]. Pariser DM, Meinking TL, Bell M, Ryan WG: Topical 0.5\% Ivermectin Lotion for Treatment of Head Lice. ; $N$ Engl J Med. 2012;367:1687-1693 